\title{
Toward next JENDL Fission Yield Data and Decay Data
}

\author{
Futoshi Minato ${ }^{1, *}$ \\ ${ }^{1}$ Nuclear Data Center, Japan Atomic Energy Agency, Tokai, Ibaraki 319-1195, Japan
}

\begin{abstract}
JENDL group has developed evaluated fission yield and decay data. They are now planned to be upgraded in order to embody new experimental data and theoretical developments obtained after the last release. In this paper, we briefly describe a plan and present activity toward next JENDL fission yield and decay data.
\end{abstract}

\section{Introduction}

Japanese Evaluated Nuclear Data Library (JENDL) keeps various kinds of evaluated nuclear data and contributes developments of nuclear physics and its applications. In the special purpose files, JENDL FP ("F"ission "P"roduct) Fission Yield Data file 2011 (JENDL/FPY2011) and JENDL FP Decay Data file 2011 (JENDL/FPD-2011) were released in 2011 [1]. They were consistently evaluated to reproduce decay heat generated from FP after thermal, fast, and $14 \mathrm{MeV}$ neutron induced fission for uranium, plutonium and thorium, using up-todate knowledge of nuclear physics at that time. Later on, in order to complement other nuclei than FP, JENDL Decay Data File 2015 (JENDL/DDF-2015) was released in 2015 [2]. The number of nuclei compiled in JENDL/FPD-2011 is 1,284, while JENDL/DDF-2015 grows into 3,287.

After the release of those evaluated nuclear data files, some issues were found in the JENDL/FPY-2011. JENDL/FPY-2011 was originally produced on the basis of England and Rider evaluation [3]. However, it was found that fission yield of ${ }^{86} \mathrm{Ge}$ in thermal neutron fission of ${ }^{235} \mathrm{U}$ was incorrectly cited from an original experimental data and an extraordinary large yield has been assigned for ${ }^{86} \mathrm{Ge}$ in England and Rider evaluation [3]. Although this problem had been already pointed out in 2002 by Oyamatsu [4], no special treatment was done when JENDL/FPY-2011 was made. This problem as well as other extraordinary large fission yields of thermal neutron induced fission of ${ }^{235} \mathrm{U}$ was corrected by using a theoretical model in the revised version of JENDL/FPY-2011 released in 2016 [5]. Simultaneously, we realize an importance of re-evaluation of fission yield and decay data because a long time has passed since England and Rider evaluation [3] was published.

There still exist issues that are not solved in the present evaluated fission yield and decay data. The first is about the summation calculation of delayed neutron yields using JENDL/FPY-2011 and JENDL/FPD-2011 (or JENDL/DDF-2015). While we paid a special attention to the summation calculations of decay heat when JENDL/FPY-2011 and JENDL/FPD-2011 were evaluated, we did not put effort in those of delayed neutron yields. As a result that we ignored delayed neutron yields in the evaluation, large deviations were

\footnotetext{
*e-mail: minato.futoshi@jaea.go.jp
} 
left between the summation calculation by JENDL/FPY-2011 and JENDL/FPD-2011 and experimental data (this will be shown in sec. 2). Due to the correction of ${ }^{86} \mathrm{Ge}$ in the revision of JENDL/FPY-2011 [5], the summation calculation of the delayed neutron yields for ${ }^{235} \mathrm{U}$ was somewhat improved. However, there still remain gaps between the summation calculation and experimental data not only for ${ }^{235} \mathrm{U}$ but also other nuclei. In addition, as a specific problem in the JENDL decay data, delayed neutron spectrum is not compiled in JENDL/FPD2011 and JENDL/DDF-2015, although it is included in ENDF/VIII.0 decay data. It is discussed that delayed neutron spectra can be used for nuclear non-destructive analysis [6]. This lack reduces potential possibilities of applications using JENDL decay data.

Besides delayed neutron yields and decay heats, a lot of observables are resulted from nuclear fission and subsequent decay of FP, for example, prompt fission $\gamma$ emission, delayed $\gamma, \beta$, and neutrino emissions. Those observables are radioactive until all fission fragment turns into stable nuclei. It is widely discussed in recent years that those observables can be applied to non-destructive analysis, monitoring nuclear reactor, etc. [7-11]. The past JENDL fission yield and decay data, however, have not paid a particular attention to those observables as well as delayed neutron yields. Next JENDL evaluated fission yield and decay data should consider those observables and be evaluated consistently and simultaneously in order to provide a more reliable evaluated data.

Since the release of JENDL/FPD-2011 and JENDL/DDF-2011, new experimental data have been obtained. In particular, half-lives and delayed neutron branching ratios have been actively measured in the last decade due to developments of radio isotope beam facilities in the world. Moreover, the total absorption gamma-ray spectroscopy (TAGS) data has been also newly measured since 2011. The TAGS data has played an important role to improve the summation calculation of decay heats when JENDL/FPY-2011 and JENDL/FPD-2011 were evaluated. We should consider those new experimental data in next JENDL evaluated fission yield and decay data.

Even though there are a plenty experimental data available, all of information on decay data is not necessarily known. To produce a completely arranged decay data file, theoretical models are used to compensate the lacks of experimental data. Gross theory and FRDM+QRPA models have been applied to JENDL and ENDF decay data, respectively. For next JENDL decay data file, we plan to make use of a microscopic model of in addition to the Gross theory used for JENDL/FPD-2011.

In this paper, we summarize a plan of next JENDL evaluated fission yield and decay data. In sec. 2.1, we describe new points to be included in the next JENDL decay data. In sec. 2.3, errors found in JENDL/FPD-2011 and JENDL/DDF-2015, which are to be corrected in next JENDL decay data are listed In sec. 2.2, two microscopic theoretical models that we are developing so far are explained and a recent activity using them is given. In sec. 3, a plan of new JENDL fission product yield data is briefly described. In sec. 4, we conclude our remarks.

\section{Improvements planned in next JENDL decay data}

In this section, we describe a main feature of next JENDL decay data. In sec. 2.1, new points to be included in next JENDL decay data are briefly described. In sec. 2.2, we explain microscopic theoretical models that are planned to be used for next JENDL decay data. In sec. 2.3, errors found in JENDL/FPD-2011 and JENDL/DDF-2015 are listed. Those will be corrected in the next JENDL decay data. 


\subsection{New points from the previous data}

We list the new points to be included in the next JENDL decay data in the following:

1. Numerical values in the decay data will be updated by the latest version of ENSDF and experimental data including newly measured TAGS data.

2. Spectra of $\beta$-ray, delayed neutron, and other light particles will be included.

3. New theoretical calculations will be applied for short-life nuclei where no experimental data exist.

4. The decay data will be benchmarked by aggregated data such as decay heat, delayed neutron yield, prompt fission gamma spectra, etc.

5. Light exotic nuclei such as ${ }^{4,5,6} \mathrm{H}$, which have been omitted in JENDL/DDF-2015, will be compiled.

As mentioned in sec.1, delayed neutron yields were not used for the benchmark test of JENDL/FPY-2011 and JENDL/FPD-2011, although a special attention was put in decay heats. Figure 1 and 2 show the delayed neutron activities as a function of time for thermal and fast neutron induced fission, respectively. The summation calculation by using JENDL/FPY2011 and JENDL/FPD-2011 is compared with Keepin's six group evaluation [12]. As seen in the figure, the summation calculations of JENDL/FPY-2011 and JENDL/FPD-2011 systematically overestimate the delayed neutron activities of Keepin's evaluations both for thermal and fast neutron induced fission in a wide range of time. We plan to improve the shortcoming of the summation calculation of delayed neutron yields as well as other observables in the next JENDL evaluated fission yield and decay data.

\subsection{Theoretical Model Applications}

As mentioned in sec. 1, theoretical models are applied in evaluating decay data where no experimental data are available. We have been developing several theoretical models to apply them to nuclear decay data. Gross theory [13-15] is one of our powerful approaches. This model gives a reasonable agreement with experimental data in half-lives, delayed neutron branching ratios, and average $\beta$ and $\gamma$ emission energies in a wide range of nuclei.

Another promising model is a quasiparticle random-phase-approximation (QRPA) on the basis of non-relativistic Skyrme energy density functional (Skyrme EDF) [16] and covariant density functional [17]. We have calculated half-lives and delayed neutron branching ratios of FPs using QRPA+Skyrme EDF [16]. We developed the model considering a finite-range proton-neutron isospin $T=0$ force. The delayed neutron branching ratio and its spectra are calculated with Hauser-Feshbach statistical model. We also incorporate the statistical model to QRPA based on the covariant density functional [17] to estimate delayed neutron branching ratio in a more realistic way. At present, we are making decay data table including half-lives, delayed neutron and fission branching ratios, average $\beta$ and $\gamma$ emission energies, emitted light particle $\left(e^{ \pm}, \bar{v}_{e}, n, \alpha\right)$ spectra, using QRPA approaches described above. The result will be submitted elsewhere.

To select the best model, results of theoretical approaches have to be compared with available experimental data. Half-lives are often used as the benchmark of theoretical models. However, we plan to take a more careful test for theoretical models by using experimental data of delayed neutron branching ratios, and its activities, emitted particle energies, and etc. We have studied the summation calculation of decay heat using JENDL/FPY-2011 and decay 

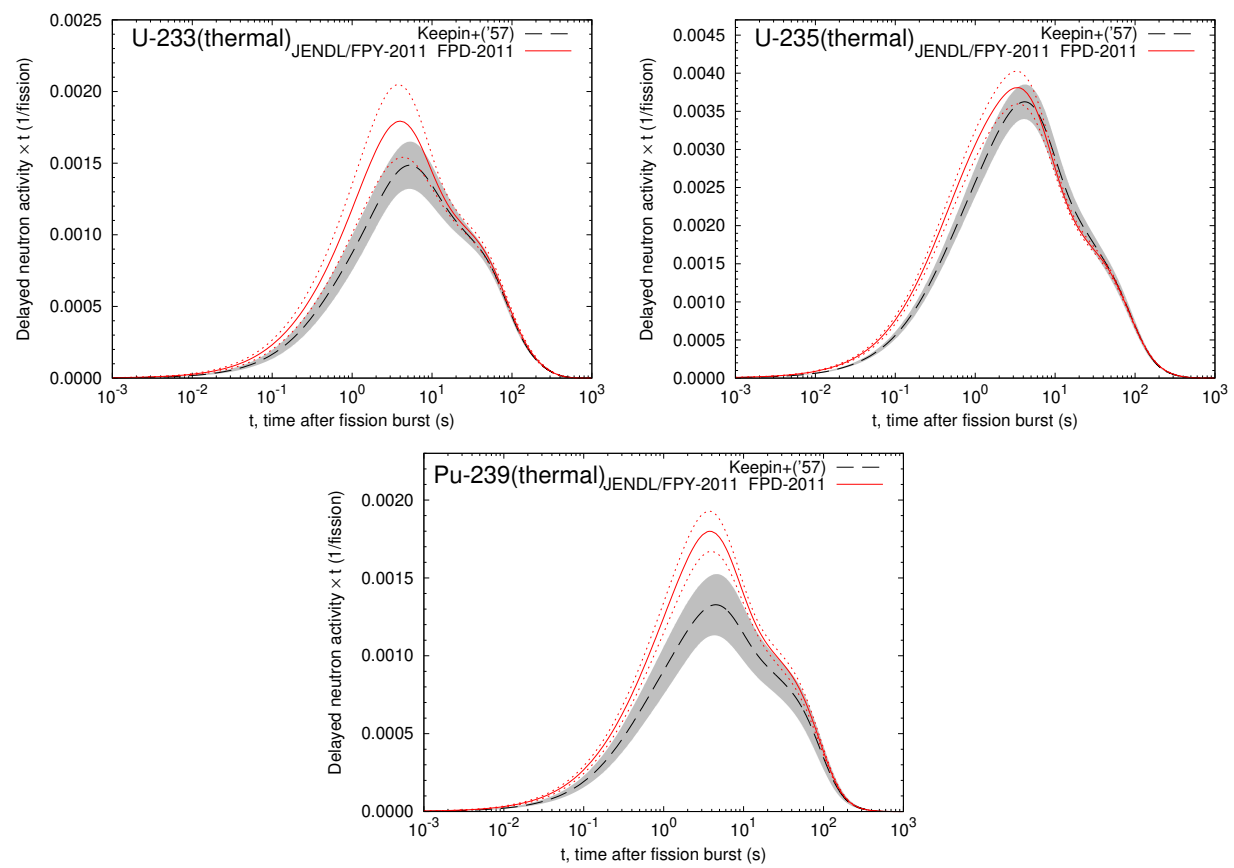

Figure 1. Delayed neutron activities for thermal neutron induced fission for ${ }^{233,235} \mathrm{U}$ and ${ }^{239} \mathrm{Pu}$ multiplied by time $(t)$. In the panels, the results of Keepin's six group evaluation [12] (the dashed line) and the summation calculation by using JENDL/FPY-2011 and JENDL/FPD-2011 (the solid line) are shown. Errors of Keepin's six group are shown by the shaded area, while the uncertainties of the summation calculation are shown with the dotted lines.

data provided purely by the above theoretical models. We found that those tests are also useful to see the best model for decay data. The result of benchmark of theoretical models will be also submitted elsewhere.

\subsection{Missing and mistaken data found in JENDL/FPD-2011}

We recognized several errors in JENDL/FPD-2011 and JENDL/DDF-2015, which are listed in the following. Those will be corrected in the next JENDL decay data. The corrected version of JENDL/FPD-2011 and JENDL/DDF-2015 are also planned to be distributed.

1. $\gamma$-ray spectra of ${ }^{241} \mathrm{Am}$ are wrong in JENDL/DDF-2015.

2. An isomeric state of ${ }^{235} \mathrm{U}$ is populated by $\alpha$ decay of ${ }^{239} \mathrm{Pu}$. However, the isomeric state of ${ }^{235} \mathrm{U}$ is missing in JENDL/DDF-2015.

3. Double $\beta^{-}$-decay $(\mathrm{RTYP}=1.1)$ is assigned for neutron deficient nucleus $\mathrm{Kr}-78$. The correct decay type is double electron capture (RTYP=2.2).

4. Two ground states are given for ${ }^{32} \mathrm{Na},{ }^{93} \mathrm{Ru},{ }^{101} \mathrm{Pd},{ }^{131} \mathrm{Pr},{ }^{137} \mathrm{Pm}$, and ${ }^{183} \mathrm{Ir}$. One of them must be an isomeric state.

5. FPs compiled in JENDL/FPY-2011 should be in JENDL/FPD-2015 as well. However, it is found that isomeric states of ${ }^{84 m} \mathrm{As},{ }^{109 m} \mathrm{Ru}$, and ${ }^{143 m} \mathrm{Xe}$ are not in JENDL/FPD2011. 

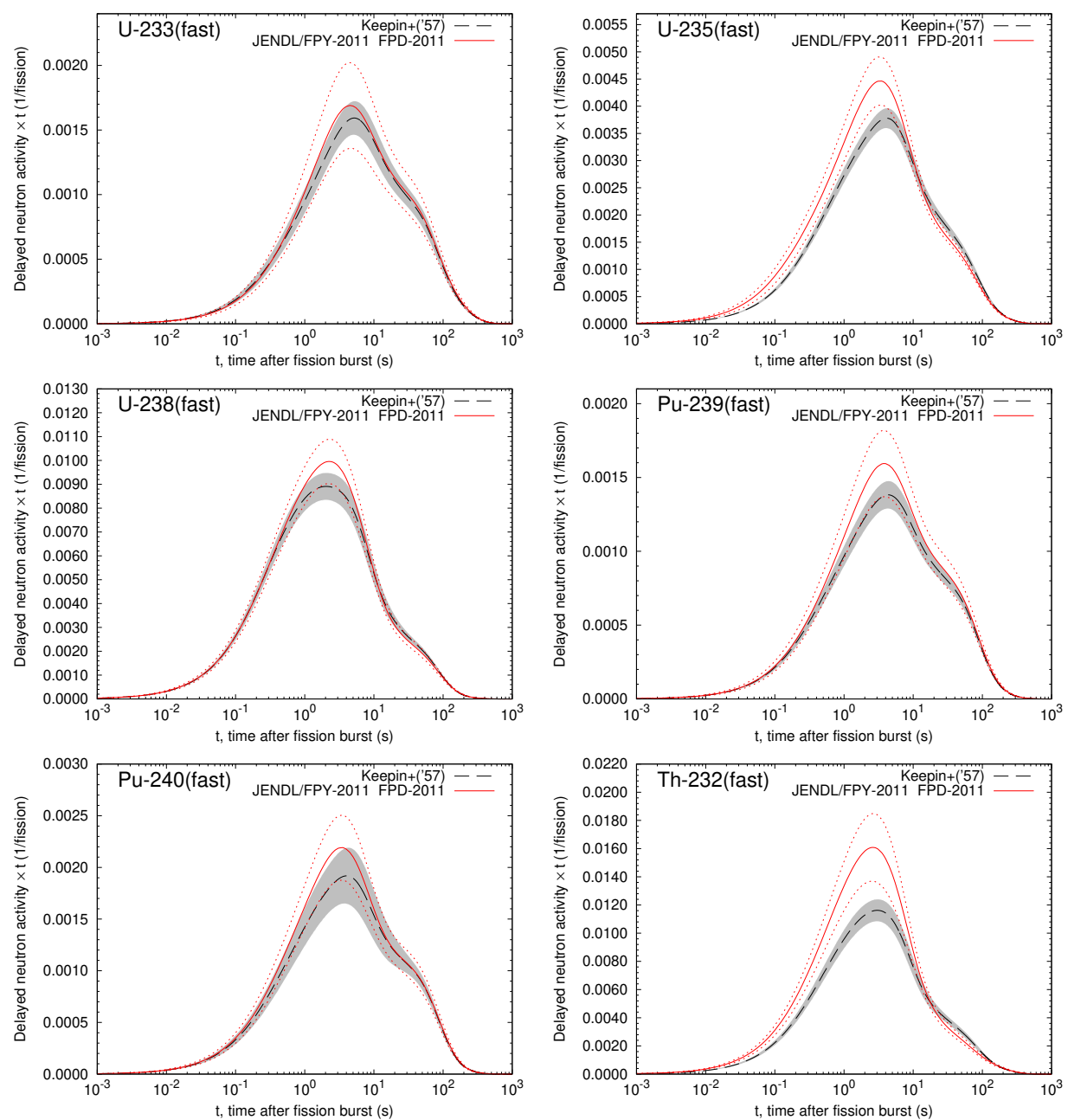

Figure 2. Same as Fig. 1, but for delayed neutron activities for fast neutron induced fission for ${ }^{233,235,238} \mathrm{U},{ }^{239,240} \mathrm{Pu}$, and ${ }^{232} \mathrm{Th}$.

6. Decay data of FPs in JENDL/FPD-2011 and JENDL/DDF-2015 should be identical. However, it is found that ${ }^{82} \mathrm{Se},{ }^{83,85} \mathrm{Sr},{ }^{96} \mathrm{Zr},{ }^{100} \mathrm{Mo},{ }^{111 m} \mathrm{Pd},{ }^{116} \mathrm{Cd},{ }^{128,130} \mathrm{Te},{ }^{134,136} \mathrm{Xe}$, ${ }^{136,138,142} \mathrm{Ce},{ }^{150} \mathrm{Nd}$, and ${ }^{151} \mathrm{Eu}$ are not identical.

7. ${ }^{109} \mathrm{Cd}$ decays to an isomeric state of ${ }^{109} \mathrm{Ag}$. However, it decays to the ground state in JENDL/FPD-2011 and JENDL/DDF-2015.

\section{Next JENDL Fission Yield}

We briefly describe an activity for new fission product yield data in this section. It is planned to adopt the evaluated fission yields produced by Tokyo institute of Technology group [18] as JENDL-5 fission yield data file. The evaluated data have several developments as compared to JENDL/FPY-2011. For example, it considers up-to-date insights on nuclear physics and is 
benchmarked by product inventory (PI) data provided from various nuclear reactors. In particular, it includes covariance data, thus it enables us to estimate a more reliable uncertainties when summation calculations are performed.

\section{4 summary}

We described a plan and recent activity for next JENDL evaluated fission yield and decay data. We are currently working to distribute them within a few years. Other than described in this paper, any request to our next evaluated data is welcomed.

\section{References}

[1] J. Katakura, JENDL FP Decay Data File 2011 and Fission Yields Data File 2011, JAEA-Data/Code 2011-025 (2011)

[2] J. Katakura, F. Minato, JENDL Decay Data File 2015, JAEA-Data/Code 2015-030 (2016)

[3] T.R. England, B.F. Rider, Evaluation and Compilation of Fission Product Yields, LAUR-94-3106, ENDF-349 (1994)

[4] K. Oyamatsu, Journal of Nuclear Science and Technology 39, 1109 (2002)

[5] J. Katakura, F. Minato, K. Ohgama, EPJ Web of Conferences 111, 08004 (2016)

[6] T. Akyurek, S. Usman, Progress in Nuclear Energy 85, 525 (2015)

[7] L.E. Smith, E.K. Mace, A.C. Misner, M.W. Shaver, IEEE Transactions on Nuclear Science 57, 2247 (2010)

[8] P.H. Liem, S. Amini, A.G. Hutagaol, T.M. Sembiring, Annals of Nuclear Energy 56, 57 (2013)

[9] M.V. Mora, A.G. Padilla, J.L.C. Palomino, L.A.A. Terremoto, Progress in Nuclear Energy 53, 344 (2011)

[10] H. Furuta, Y. Fukuda, T. Hara, T. Haruna, N. Ishihara, M. Ishitsuka, C. Ito, M. Katsumata, T. Kawasaki, T. Konno et al., Nuclear Instruments and Methods in Physics Research Section A: Accelerators, Spectrometers, Detectors and Associated Equipment 662, 90 (2012)

[11] F. Suekane, Nuclear Physics B - Proceedings Supplements 235-236, 33 (2013), the XXV International Conference on Neutrino Physics and Astrophysics

[12] G.R. Keepin, T.F. Wimett, R.K. Zeigler, Phys. Rev. 107, 1044 (1957)

[13] M. de Saint Simon, O. Sorlin, eds., Exotic nuclei and atomic masses. Proceedings, International Conference, ENAM'95, Arles, France, June 19-23 (1995)

[14] T. Yoshida, T. Tachibana, J. Nucl. Sci. Technol. 37, 491 (2000)

[15] T. Tachibana, RIKEN Review, Focused on Models and Theories of the Nuclear Mass 26, 109 (2000)

[16] F. Minato, EPJ Web of Conferences 122, 10001 (2016)

[17] T. Marketin, L. Huther, G. Martinez-Pinedo, Phys. Rev. C 93 (2016)

[18] K. Tsubakihara et al. (to be submitted) 